\title{
Gasification effects in the heterogeneous ignition of a condensed fuel by a hot gas $\dagger$
}

\author{
M. KINDELÁN AND A. LIÑ Á Nł
}

\begin{abstract}
An analysis is presented to describe the heterogeneous ignition of a condensed fuel suddenly exposed to a hot oxidizing atmosphere. The exothermic heterogeneous reaction, generating gaseous products, is considered to be of the Arrhenius type with an activation energy large compared with the initial thermal energy of the fuel. Instantaneously after contact with the gases the surface temperature rises to a jump value which is calculated allowing for variable transport properties of fuel and gas. The effect of the chemical heat release and the cooling effect due to the gasification flow are taken into account in obtaining an integral equation, involving a single parameter $\Delta$, which is solved to describe the evolution of surface temperature with time. A runaway in surface temperature is found to occur at a well defined ignition time, which is calculated as a function of $\Delta$. For values of $\Delta$ above a critical value no ignition occurs because the cooling effects of the gasification flow dominate over the effects of chemical heat release.
\end{abstract}

\section{Introduction}

DIFFERENT theories have been proposed to explain the ignition mechanism of a condensed material. These theories can be classified as condensed-phase, gasphase and heterogeneous ignition theories according to the location of the reaction responsible for ignition. Experimental results backing each of these locations have been presented in the past.

In this paper we develop an asymptotic analysis of ignition of a condensed material which is suddenly exposed to a hot ambient gas. These conditions occur, for instance, when a shock wave is reflected by a condensed material located at the end of a shock tube. We will consider the case in which an exothermic heterogeneous reaction takes place at the surface of the material, generating gaseous products.

Anderson et al. (1964) proposed the heterogeneous ignition mechanism trying to explain experimental evidence showing the feasibility of igniting a condensed fuel by contact with a powerful oxidizer (hypergol). An analytical description of this model was carried out by Williams (1966), using Laplace transforms to obtain an integral equation relating the surface temperature to time. Although the formulation of the problem included a flux of radiant energy absorbed at the surface, only the case of zero flux was numerically integrated. For large 
activation energies the numerical results exhibited "runaway in surface temperature" at a finite time (ignition time).

Williams' analysis has been generalized to include the effects of in-depth absorption of radiation (Bradley and Williams, 1970) and unequal initial temperatures (Waldman and Summerfield, 1969) in the solid and gas phases (shock tube conditions).

Closed form expressions for the ignition time as function of the parameters entering the problem, have been derived by Waldman (1970) using both a local similarity hypothesis and an adaption of a method proposed by Rae.

Grishin and Ignatenko (1971), calculated the first three terms of an expansion of the surface temperature in powers of the square root of time. The resulting surface temperature history exhibits an inflection point at a time which they define as the ignition time. However the expansion for the surface temperature does not converge for times close to the time of thermal runaway.

Yung and Chung (1974) used the Laplace method for large activation energy in an incorrect way to obtain an ordinary differential equation describing the surface temperature evolution with time. By numerically integrating this equation, the authors obtain approximate values of the ignition time, although the analysis is not asymptotically correct.

Liñán and Crespo (1972) have analyzed the heterogeneous ignition process, under radiant and shock tube conditions, in the limit of high activation energy. They derive an integral equation for the surface temperature involving a single radiant flux parameter; the solution exhibited thermal runaway at a finite time.

The present paper generalizes the results of the preceding analyses for ignition under shock tube conditions by including the effects of convection that were neglected in all of them. It is shown that in general the convective effects are important in heterogeneous shock-tube ignition, and therefore they should not be neglected, without justification.

Upon contact with the hot gas, the surface temperature rises instantaneously to an intermediate "jump temperature" which is a function of the initial temperatures in the solid and gas and of the ratio of thermal responsivities. In absence of chemical reaction, the surface temperature remains constant and the width of the conduction layer increases with the square root of time. However, in the presence of a surface reaction there are two effects that tend to modify the temperature at the surface. First, because of the gasification flow the hot gases are blown away from the surface and cold condensed material is brought towards the surface thereby decreasing the surface temperature. Second, the energy released by the exothermic reaction has the effect of raising the surface temperature.

Two different regimes are observed depending on which of these two competitive effects dominates. If the effect of the convective flow dominates the surface temperature continuously decreases while the condensed material gasifies. The surface regression rate is maximum at the time of contact with the hot gas, and thereafter decreases with time approaching zero for long times. If the effect of heat released by the reaction dominates, the surface temperature increases with time thereby accelerating the reaction rate. This selfaccelerating 
behaviour causes thermal runaway of the surface temperature at a finite ignition time.

In the following analysis we derive an integral equation with a single parameter, to describe the time evolution of the surface temperature. This parameter is a measure of the effects of convection and has a critical value which separates the two different regimes.

\section{Formulation}

We consider a one dimensional model with the solid occupying the half space $x<0$ and the oxidizer-containing gas the region $x>0$.

Figure 1 is a schematic representation of the process and shows the effects accounted for in this study.

It is assumed that an Arrhenius heterogeneous reaction, of arbitrary order with respect to oxidizer mass fraction, occurs at the interface between the condensed fuel and the ambient oxidizer. For convenience the origin is fixed at the surface of the regressing material and the mass coordinate $\psi$ ia introduced as the new space coordinate in the gas phase. We will use the well justified assumptions that the work associated with viscous, pressure and external forces is negligible. We use Fick's law to calculate the diffusion velocities and consider the specific heats to be constant in each phase. It is also assumed that the density and thermal conductivities in the condensed phase as well as their product in the gas phase are constant. Under these conditions the equations of conservation of energy in the solid and gas phases, and the oxidizer concentration equation are respectively

$$
\rho c \frac{\partial T}{\partial t}+\rho v c \frac{\partial T}{\partial x}=\frac{\partial}{\partial x}\left(\lambda \frac{\partial T}{\partial x}\right)
$$

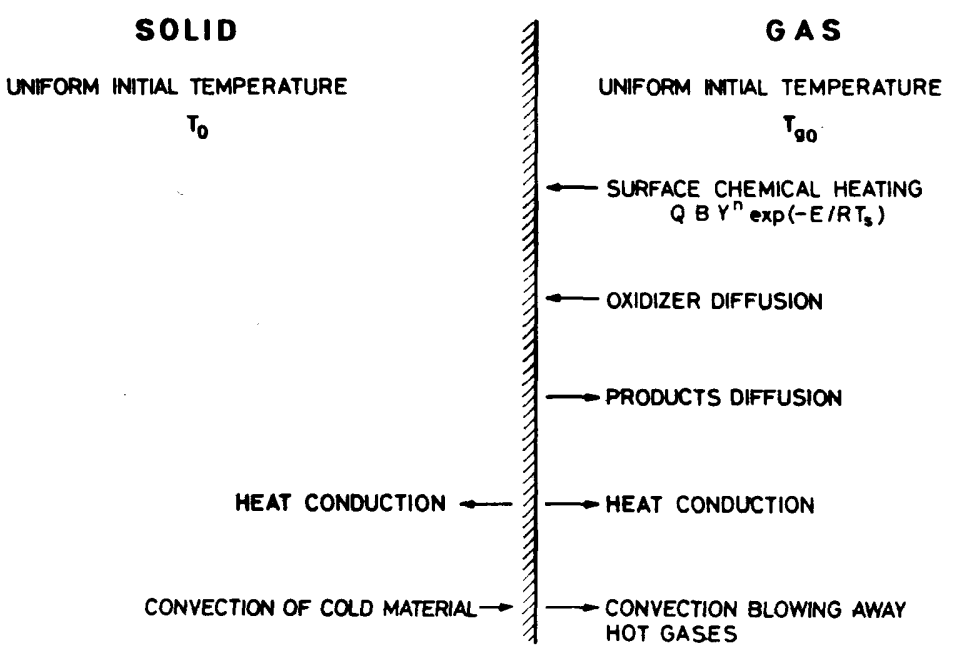

Fig. 1. Schematic representation of the process analyzed. 


$$
\begin{gathered}
c_{g} \frac{\partial T_{g}}{\partial t}+\rho v c_{g} \frac{\partial T_{g}}{\partial \psi}=\frac{\partial}{\partial \psi}\left(\rho_{g} \lambda_{g} \frac{\partial T_{g}}{\partial \psi}\right) \\
\frac{\partial Y}{\partial t}+\rho v \frac{\partial Y}{\partial \psi}=\frac{\partial}{\partial \psi}\left(\rho_{g}^{2} D_{g} \frac{\partial Y}{\partial \psi}\right)
\end{gathered}
$$

where all the symbols are defined in the nomenclature. The initial and boundary conditions are

$$
\begin{gathered}
T(0, x)=T(t,-\infty)=T_{0}, T_{g}(0, \psi)=T_{g}(t, \infty)=T_{g 0} \\
T(t, 0)=T_{g}(t, 0) \\
Y\left((0, \psi)=Y(t, \infty)=Y_{\infty}\right. \\
-\left.\lambda \frac{\partial T}{\partial x}\right|_{s}+\left.\rho_{g} \lambda_{g} \frac{\partial T_{g}}{\partial \psi}\right|_{s}=\rho v\left[\left(c_{g}-c\right)\left(T_{s}-T_{1}\right)-Q\right] \\
\rho v\left(Y_{s}+m\right)=\left.\rho_{g}{ }^{2} D_{g} \frac{\partial Y}{\partial \psi}\right|_{s}
\end{gathered}
$$

where the surface regression rate $v$ is given by

$$
v=B Y_{s}^{n} \exp \left(-E / R T_{s}\right)
$$

and $m$ is the mass stoichiometric coefficient in the reaction

$$
\{F\}+m\{O\} \rightarrow \text { gaseous products }
$$

At time zero the temperature in the gas phase is suddenly raised to a higher value $T_{g 0}$. For early times the temperature gradients at the surface are very large, and therefore the right hand side of eqn (6) is negligible. Under these conditions the problem is identical to the heating of an inert condensed material under shock tube conditions, whose solution is well known in the case of constant values of density, heat capacity and conductivity. We derive in an appendix the solution to the inert problem for arbitrary dependence of the thermal properties on temperature. In the analysis that follows, the quantity $\rho_{g} \lambda_{g}$ is taken as constant, but its value is chosen in such a way that the correct "jump temperature" (temperature at the surface with inert heating, derived in the appendix) is obtained when the inert problem is solved in the assumption of constant thermal properties.

Introducing the nondimensional variables

$$
\theta=\frac{T-T_{1}}{T_{g 0}-T_{1}}, \quad \theta_{g}=\frac{T_{g}-T_{1}}{T_{g 0}-T_{1}}
$$




$$
\xi=\left\{\begin{array}{l}
\frac{Q}{T_{g 0}-T_{1}} \frac{\rho v_{1}}{\rho_{g} \lambda_{g}} \frac{\Gamma}{1+\Gamma} \psi \quad \text { in the gas phase } \\
-\frac{\rho v_{1} Q}{T_{g 0}-T_{1}} \sqrt{\left(\frac{\rho c}{\rho_{g} \lambda_{g} c_{g} \lambda}\right) \frac{\Gamma}{1+\Gamma} x \quad \text { in the solid phase }} \\
\tau=\left(\frac{Q}{T_{g 0}-T_{1}}\right)^{2} \frac{\left(\rho v_{1}\right)^{2}}{\rho_{g} \lambda_{g} c_{g}}\left(\frac{\Gamma}{1+\Gamma}\right)^{2} t
\end{array}\right.
$$

where $\Gamma=\sqrt{ }\left((\rho \lambda c)_{g} /(\rho \lambda c)\right), T_{1}$ is the jump temperature

$$
T_{1}=T_{0}+\left(T_{80}-T_{0}\right) \frac{\Gamma}{1+\Gamma}
$$

and $v_{1}$ is the value of $v$ for $T_{s}=T_{1}$ and $Y_{s}=Y_{\infty}$, eqns (1)-(7) become

$$
\begin{gathered}
\frac{\partial \theta}{\partial \tau}-\alpha(1+\Gamma) \frac{v}{v_{1}} \frac{\partial \theta}{\partial \xi}=\frac{\partial^{2} \theta}{\partial \xi^{2}} \\
\frac{\partial \theta_{g}}{\partial \tau}+r \alpha \frac{1+\Gamma}{\Gamma} \frac{v}{v_{1}} \frac{\partial \theta_{g}}{\partial \xi}=\frac{\partial^{2} \theta_{g}}{\partial \xi^{2}} \\
\frac{\partial Y}{\partial \tau}+r \alpha \frac{1+\Gamma}{\Gamma} \frac{v}{v_{1}} \frac{\partial Y}{\partial \xi}=\frac{1}{L e} \frac{\partial^{2} Y}{\partial \xi^{2}} \\
\theta(0, \xi)=\theta(\tau, \infty)=-\Gamma, \quad \theta_{g}(0, \xi)=\theta_{g}(\tau, \infty)=1 \\
\theta(\tau, 0)=\theta_{g}(\tau, 0) \\
Y(0, \xi)=Y(\tau, \infty)=Y_{\infty} \\
\left.\frac{\partial \theta}{\partial \xi}\right|_{s}+\left.\Gamma \frac{\partial \theta_{g}}{\partial \xi}\right|_{s}=-(1+\Gamma) \frac{Q-\left(T_{s}-T_{1}\right)\left(c_{g}-c\right)}{Q} \frac{v}{v_{1}} \\
\left.\frac{\partial Y}{\partial \xi}\right|_{s}=\left(Y_{s}+m\right) \alpha r L e \frac{1+\Gamma}{\Gamma} \frac{v}{v_{1}}
\end{gathered}
$$

where

$$
v / v_{1}=\left(Y_{s} / Y_{\infty}\right)^{n} \exp \left(-E / R T_{s}+E / R T_{1}\right) .
$$

\section{Analysis of the ignition process}

Upon contact with the hot gases, the surface temperature will be instantaneously raised to its jump value. However, the exothermic reaction at the surface introduces two effects that modify the jump temperature. On the one hand, the gasification flow brings cold material towards the surface and pushes the hot gases away, thus reducing the surface temperature. On the other hand the heat released by the exothermic reaction causes an increase in surface temperature. Ignition will or will not occur depending on which effect dominates. 
It is observed that for large values of the nondimensional activation energy $E / R T_{1}$, small increments in surface temperature of order $R T_{1}{ }^{2} / E$ produce an increment in the reaction rate by a factor $e$.

The corresponding increments of $\theta$ and $\theta_{g}$ from their inert values

$$
\theta_{I g}=-\theta_{I} / \Gamma=\operatorname{erf}(\xi / 2 \vee \tau)
$$

are small, of order $R T_{1}^{2} /\left\{E\left(T_{g 0}-T_{1}\right)\right\}$, so that it is convenient to introduce the nondimensional variables

$$
\begin{gathered}
\chi=\beta\left(\theta-\theta_{I}\right) / \Gamma, \quad \chi_{g}=\beta\left(\theta_{g}-\theta_{I_{g}}\right) / \Gamma \\
y=\beta\left(Y_{\infty}-Y\right) /\left\{\left(Y_{\infty}+m\right) \alpha r(1+\Gamma) \bigvee L e\right\} \\
\sigma=\beta^{2} \tau / \Gamma^{2}, \quad z=\beta \xi / \Gamma
\end{gathered}
$$

where

$$
\beta=E\left(T_{1}-T_{0}\right) / R T_{1}^{2}
$$

is a nondimensional activation energy. The nondimensional time $\sigma$ and fuel mass fraction $y$ have been defined so that they are of order unity during the ignition transient. The analysis that follows is based on the assumption that the parameter $\beta$ is large compared to unity which is equivalent to requiring that the reaction rate at the jump temperature be much faster than the gasification rate at the initial temperature. Thus, it is necessary to assume that $\beta$ is large in order to have a physically realistic problem in which a non reacting condensed material is suddenly brought to vigorous reaction upon contact with a hot oxidizer-containing gas. The ratio of thermal responsivities $\Gamma$ is usually small, but it may become of order unity for large values of pressure or $T_{g 0}$, since it is proportional to the square root of $\rho_{g} \lambda_{g}$. Thus, the parameter $\beta / \Gamma$ is at least of order $\beta$.

Introducing the nondimensional variables (22)-(24) and taking the limit $\beta \rightarrow \infty$, eqns (14)-(20) simplify to

$$
\begin{gathered}
\frac{\partial \chi}{\partial \sigma}-\frac{\partial^{2} \chi}{\partial z^{2}}=-\frac{P}{\sqrt{ }(\pi \sigma)} \exp \left(-\frac{z^{2}}{4 \sigma}+\chi_{s}\right) \\
\frac{\partial y}{\partial \sigma}-\frac{1}{L e} \frac{\partial^{2} y}{\partial z^{2}}=0 \\
\chi(0, z)=\chi(\sigma, \infty)=y(0, z)=y(\sigma, \infty)=0 \\
\chi(\sigma, 0)=\chi_{g}(\sigma, 0) \\
\left.\frac{\partial \chi}{\partial z}\right|_{s}+\left.\Gamma \frac{\partial \chi_{g}}{\partial z}\right|_{s}=-(1+\Gamma) \exp \left(\chi_{s}\right) \\
\left.\frac{\partial y}{\partial z}\right|_{s}=-\sqrt{ } L e \exp \left(\chi_{s}\right) .
\end{gathered}
$$


The parameter $P$ appearing in eqn (26), describing the temperature in the condensed phase, equals $\alpha \Gamma(1+\Gamma)$. The equation that describes the gas-phase temperature is identical to eqn (26) with $P$ replaced by $\alpha r(1+\Gamma) / \Gamma$.

Only the gradient of the inert temperature appears in the term describing the effect of convection, given by the right-hand side of eqn (26), and no convective effects appear in eqn (27) describing the mass fraction. It should also be observed that in the limit $\beta \rightarrow \infty$ the equations and boundary conditions describing the temperature distributions are uncoupled from those describing the mass fraction. Green functions may be used to write the solution to eqns (26), (28) and (29) as

$$
\begin{aligned}
\chi(\sigma, z)=- & \frac{P}{2 \pi} \int_{0}^{\sigma} \frac{\exp \left(\chi_{s}^{\prime}\right)}{\sqrt{\left(\sigma^{\prime}\left(\sigma-\sigma^{\prime}\right)\right)}} \int_{0}^{\infty} \exp \left(-z^{\prime 2} / 4 \sigma^{\prime}\right) G\left(z, \sigma ; z^{\prime}, \sigma^{\prime}\right) \mathrm{d} z^{\prime} \mathrm{d} \sigma^{\prime} \\
& +\int_{0}^{\sigma} \frac{\exp \left\{-z^{2} / 4\left(\sigma-\sigma^{\prime}\right)\right\}}{\sqrt{\left(\pi\left(\sigma-\sigma^{\prime}\right)\right)}}\left(-\left.\frac{\partial \chi^{\prime}}{\partial z}\right|_{s}\right) \mathrm{d} \sigma^{\prime}
\end{aligned}
$$

where

$$
G\left(z, \sigma ; z^{\prime}, \sigma^{\prime}\right)=\exp \left\{-\left(z-z^{\prime}\right)^{2} / 4\left(\sigma-\sigma^{\prime}\right)\right\}+\exp \left\{-\left(z+z^{\prime}\right)^{2} / 4\left(\sigma-\sigma^{\prime}\right)\right\}
$$

Evaluating eqn (31) at the surface and using eqn (29) an integral equation defining $\chi_{s}$ is obtained, viz.,

$$
\chi_{s}=\int_{0}^{\sigma} \frac{\exp \left(\chi_{s}^{\prime}\right)}{\sqrt{\left(\pi\left(\sigma-\sigma^{\prime}\right)\right)}} \mathrm{d} \sigma^{\prime}-\frac{\Delta}{\sqrt{ }(\pi \sigma)} \int_{0}^{\sigma} \exp \left(\chi_{s}{ }^{\prime}\right) \mathrm{d} \sigma^{\prime}
$$

This equation describes the evolution with time of the surface temperature as a function of the single parameter $\Delta=\alpha(r+\Gamma)$, which is a measure of the cooling effect of the gasification flow. The range of values of $\Delta$ is large; when the overall reaction responsible for ignition has a small exothermicity $Q, \Delta$ may become of order unity.

Equations (27), (28), (30) may be used to obtain through an analogous procedure, an integral equation relating the surface mass fraction of oxidizer to time, viz.,

$$
y_{s}=\int_{0}^{\sigma} \frac{\exp \left(\chi_{s}^{\prime}\right)}{V\left(\pi\left(\sigma-\sigma^{\prime}\right)\right)} \mathrm{d} \sigma^{\prime} .
$$

The first term in the right hand side of eqn (33) accounts for the heat released by the exothermic surface reaction, and it is the only one appearing when the convective effects are neglected (Waldman and Summerfield, 1969). However, to neglect the convective effects is appropriate only when $\Delta$ is small; that is when the nondimensional heat of reaction $1 / \alpha$ is large compared to unity, or when both the ratio of heat capacities $r$ and the ratio of thermal responsivities $\Gamma$ are small compared to one. 
Since in eqn (33), the term associated with the effect of heat release by the reaction tends to increase the surface temperature, while the term associated with the effect of the gasification flow tends to decrease it, different regimes are observed depending on which of these two competitive effects dominates. There is a critical value $\Delta_{c}=2$ such that for $\Delta>2$ the effect of the gasification flow dominates and therefore the surface temperature continuously decreases while the hot gases are blown away from the surface. For $\Delta<2$ the effect of heat released by the reaction dominates, leading to a selfacceleratory reaction rate that causes runaway in surface temperature at a finite ignition time. For $\Delta=2$ the surface temperature remains constant at its jump value, and the temperature distribution is given by

$$
\chi=-\frac{P z}{2} \operatorname{erfc}\left(\frac{z}{2 \sqrt{\sigma}}\right)
$$

When $\Delta>2$ the problem is similar to that of the gasification of a condensed material which reacts endothermically at the surface with the ambient oxidizer. This problem has been analyzed by Kindelán and Liñán (1976), who derive an integral equation similar to (33). For very long times such that $\chi_{s}$ becomes of order $\beta$, the complete convective effects have to be retained and the Arrhenius exponent can not be linearized. This regime is also analyzed by Kindelán and Liñan (1976), who derive a similarity solution, thus completing the analysis of the gasification history. When $\Delta<2$, a finite difference version of eqn (33) has been numerically integrated through a procedure paralleling that used by Liñan and Williams (1971). The results of such integrations are shown in Fig. 2 for several values of $\Delta$. In Fig. 3, the nondimensional ignition time $\sigma_{\text {ign }}$ is plotted as a

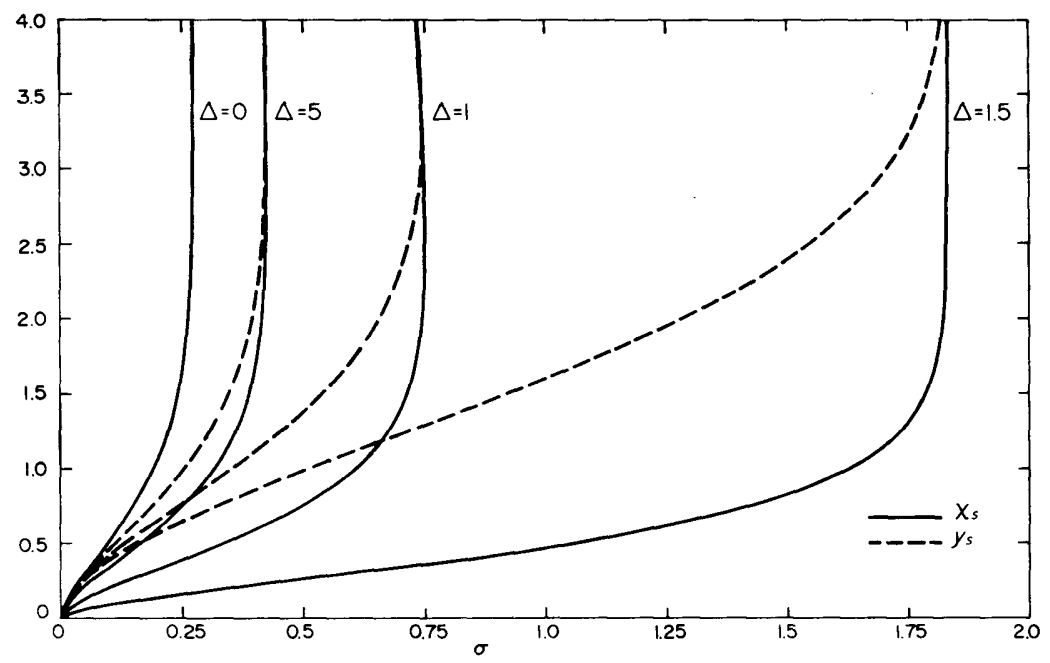

Fig. 2. Surface temperature histories, for different values of the convective parameter $\Delta$. 


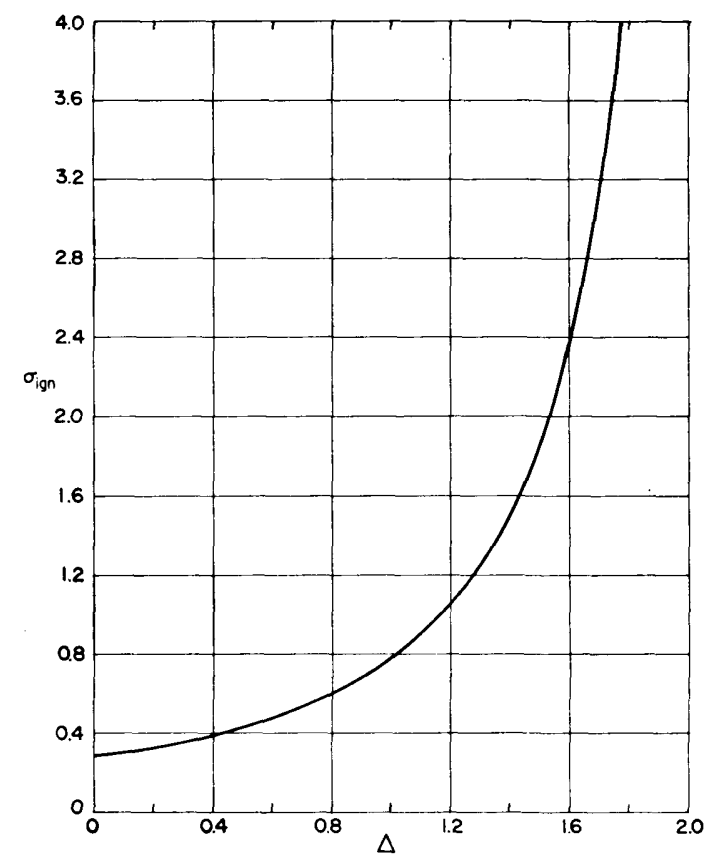

Fig. 3. Nondimensional ignition time as a function of the parameter $\Delta$.

function of $\Delta$. When $\Delta$ approaches its critical value, the ignition time tends to infinity.

For small times eqn (33) yields

$$
\chi_{s}=\sqrt{ }(\sigma / \pi)(2-\Delta)+\sigma\{1-\Delta / 2+2 \Delta(\Delta-2) / 3 \pi\}+\ldots
$$

This expansion has been previously derived (Grishin and Ignatenko, 1971) for the case $\Delta=0$.

Equation (34) has also been numerically integrated to produce the time evolution of the oxidizer mass fraction at the surface. These results are shown in Fig. 2.

\section{Discussion}

We have carried out an asymptotic analysis of the heterogeneous ignition of a condensed material, including the cooling effects of convection. The lowest order solution for the surface temperature deviation from its inert value, in an expansion in powers of the nondimensional activation energy $\beta$, is obtained by solving an integral equation. A single parameter appears in this equation to measure the effects of convection, and when these effects are dominant it is found that the gasification flow moves the region of hot gases away from the surface and no ignition occurs. There is a critical value, $\Delta_{c}=2$, of the convective parameter $\Delta$ that separates conditions under which ignition occurs, from condi- 
tions in which the surface temperature continuously decreases with time. The existence of these critical conditions has not been previously suggested, since this criticallity is associated with convective effects and previous analyses have neglected these effects.

For values of $\Delta<2$, there is a thermal runaway at an ignition time $t_{\mathrm{ign}}=$ $t_{c} \sigma_{\text {ign }}(\Delta)$ : the function $\sigma_{\text {ign }}(\Delta)$ is represented in Fig. 3, and

$$
t_{c}=\frac{R^{2} T_{1}^{4}(1+\Gamma)^{2} \lambda c \exp \left(2 E / R T_{1}\right)}{Q^{2} Y_{\infty}^{2 n} B^{2} E^{2} \rho}
$$

where $T_{1}$ is the jump temperature defined in eqn (13). The characteristic time is inversely proportional to the ambient oxidizer mass fraction raised to twice the reaction order of the heterogeneous reaction. The dependence on the initial gas temperature is mainly exhibited through the term $\exp \left(2 E / R T_{1}\right)$ in the definition of $t_{c}$. Figure 4 shows explicitly the dependence of the ignition time on the initial gas temperature for all other parameters fixed. It is observed that the ignition time decreases rapidly with increasing initial gas temperature. However, when this initial temperature is high enough as to make the parameter $\Delta$ close to 2 , it is seen from Fig. 3 that the nondimensional ignition time $\sigma_{\text {ign }}$ approaches infinity. In Fig. 4 it is observed that for high values of $T_{80}, t_{\mathrm{ign}}$ increases with the initial gas temperature approaching infinity when $\Delta$ goes to 2 .

Our analysis also shows that for large activation energy, the change in oxidizer mass fraction from its initial value is small and therefore it can be neglected when analyzing the ignition process.

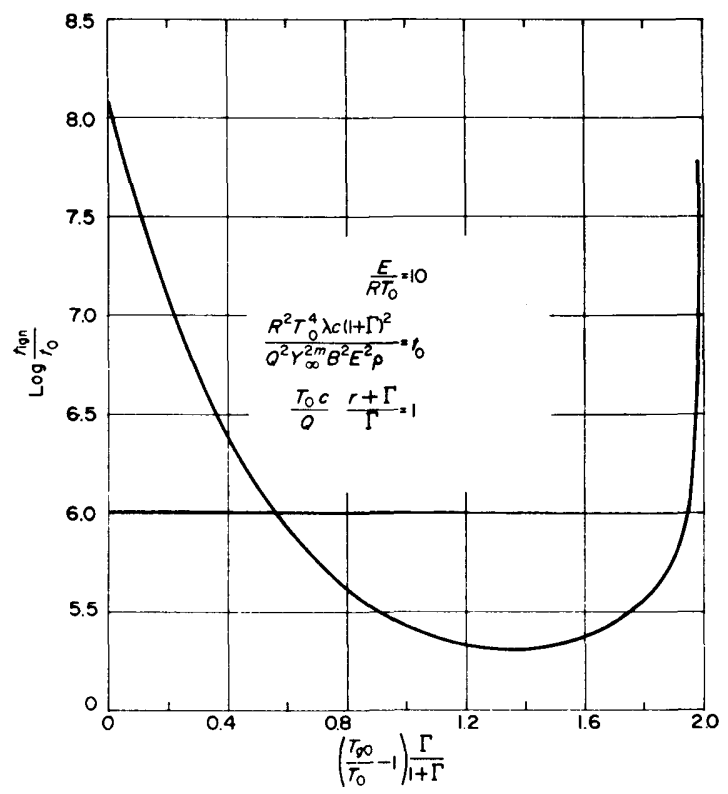

Fig. 4. Ignition time as a function of the initial gas temperature. 
This study is also applicable to the case in which an endothermic surface reaction produces a gaseous fuel which reacts with the ambient oxidizer immediately after gasification. In this case the heat of reaction $Q$ should be replaced by the difference between the heat of reaction and the heat of gasification, as discussed by Kindelán and Liñán (1976).

Acknowledgements - This research has been sponsored in part by the Air Force Office of Scientific Research, through the European Office of Aerospace Research, OAR, United States Air Force, under Grant AFOSR No. 73-2535.

\section{References}

Anderson, R., Brown, R.S. and Shannon, L. J. (1964) Heterogeneous reaction in ignition and combustion of solid propellants. AIAA J. 2, 179-180.

Bradley, H. H. Jr. and Williams, F. A. (1970) Theory of radiant and hypergolic ignition of solid propellants. Comb. Sci. Tech. 2, 41-52.

Grishin, A. M. and Ignatenko, N. A. (1971) Hybrid-propellant ignition by heterogeneous reaction. Fiz. Goreniya i Vzryva 7, 510-518.

Hansen, C. F. (1965), Effects of variable thermal properties in one-dimensional heat flow. Phys. Fluids 8, 2288-2289.

Kindelán, M. and Liñán, A. (1976) Gasification of a condensed material in a hot gas. AIAA paper $76 / 671$.

Liñán, A. and Williams, F. A. (1971) Theory of ignition of a reactive solid by constant energy flux. Comb. Sci. Tech. 3, 91-98.

Liñán, A. and Crespo, A. (1972) An asymptotic analysis of radiant and hypergolic ignition of solid propellants. Comb. Sci. Tech. 6, 223-232.

Waldman, C. H. and Summerfield, M. (1969) Theory of propellant ignition by heterogeneous reaction. AIAA J. 7, 1359-1361.

Waldman, C. H., Cheng, S. I., Sirignano, W. A. and Summerfield, M. (1969) Theoretical studies of diffusion flame structures. A. M. S. Rep. No. 860, Department of Aerospace and Mechanical Sciences, Princeton University.

Waldman, C. H. (1970) Theory of heterogeneous ignition. Comb. Sci. Tech. 2, 81-93.

Williams, F. A. (1966) Theory of propellant ignition by heterogeneous reaction. AIAA J. 4, $1354-1357$.

Yung, S. C. and Chung, P. M. (1974) Asymptotic and numerical solutions of certain heterogeneous ignition problems, Proc. of the 1974 Heat Transfer and Fuid Mechanics Institute, pp. 136-153, Stanford University Press.

\section{Appendix}

The ignition time of a condensed fuel under shock tube conditions has been found to depend strongly on the "jump" temperature. Therefore, it is important to compute correctly its value, taking into account the dependence of the thermal properties on temperature.

Hansen (1965) analyzed the effect of variable thermal properties in one-dimensional heat flow and derived the dimensionless heat flux at the boundary as a function of the surface temperature, in the case where the density, heat capacity and thermal conductivity are proportional to powers of temperature.

The jump temperature is the surface temperature instantaneously established after contact of a condensed material with a hot ambient gas. Waldman et al. (1969) analyzed this problem and derived the "jump" temperature by assuming the Chapman-Rubesin approximation. In this appendix, Hansen's analysis is used to derive the jump temperature with arbitrary temperature dependence of the density and thermal conductivity in the gas-phase.

The equations describing the process are eqns (1), (2), (4), (6), (8) with $v=0$. These equations describe the temperature distribution in the absence of surface reaction and gasification. No 
characteristic length or time appears in these equations; therefore it is easy to show by dimensional analysis arguments that the equations admit a similarity solution, with the surface or jump temperature $T_{1}$ remaining constant for $t>0$. Therefore the inert solution in the solid is still given by eqn (21) if its density specific heat and conductivity are constant; in the gas-phase the energy equation, when written in terms of $\theta=T_{g} / T_{1}$ and the similarity variable

$$
\eta=-\psi\left[c_{\mathrm{g}} / 4 t\left(\rho_{\mathrm{g}} \lambda_{\mathrm{g}}\right)_{1}\right]^{1 / 2}
$$

takes the form

$$
\frac{\partial}{\partial \eta}\left\{\frac{\rho_{g} \lambda_{g}}{\left(\rho_{g} \lambda_{g}\right)_{1}} \frac{\partial \theta}{\partial \eta}\right\}+2 \eta \frac{\partial \theta}{\partial \eta}=0
$$

where the term $-4 t(\partial \theta / \partial t)$ has been left out of the equation, because $\theta$ is only a function of $\eta$. The specific heat in the gas phase is taken as constant. The surface boundary conditions are $\theta(0)=1$ and

$$
\left.\left.\frac{\partial \theta}{\partial \eta}\right|_{\eta=0}=\frac{2}{\sqrt{ } \pi}\left(1-\frac{T_{0}}{T_{1}}\right) \sqrt{\left(\frac{\rho \lambda c}{\left(\rho_{g} \lambda_{g} c_{g}\right)_{1}}\right.}\right)
$$

obtained from eqn (6). At infinity $\theta(\infty)=T_{80} / T_{1}$.

To calculate $T_{1}$ and the solution of eqn (A2) for a given dependence of $\rho_{g} \lambda_{g}$ on temperature we proceed as follows. We can integrate eqn (A2) to calculate $\theta(\infty)=T_{g 0} / T_{1}$ in terms of $(\partial \theta / \partial \eta)_{\eta=0}$ or equivalently $T_{1}$ if eqn (A3) is taken into account. The relation $T_{g 0}=T_{80}\left(T_{1}\right)$ enables us to calculate $T_{1}$ for given $T_{80}$.

For the particular case in which $\rho_{g} \lambda_{g}$ is assumed to be proportional to the temperature raised to an arbitrary power $w$.

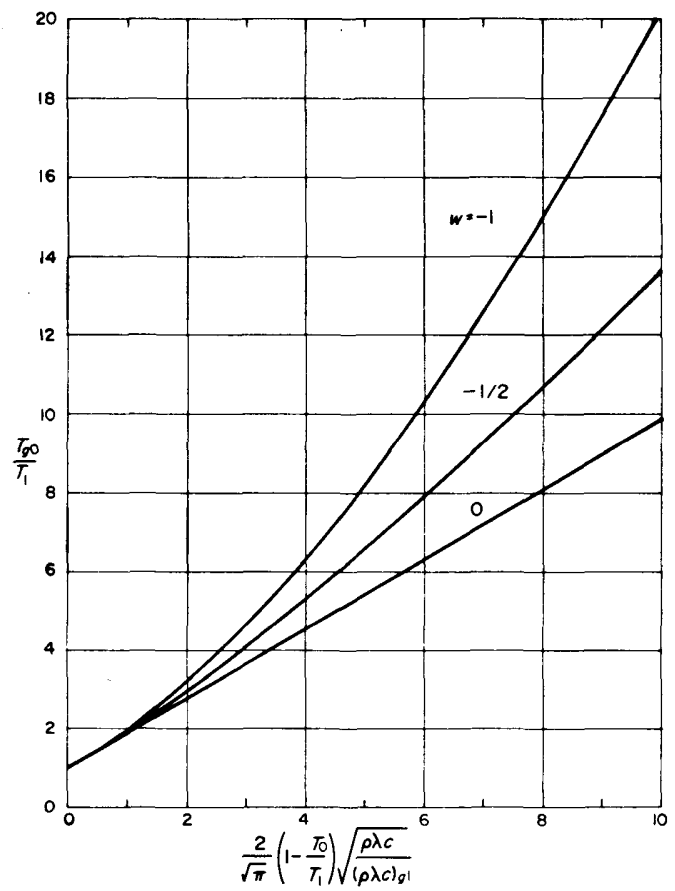

Fig. 5. Ratio $T_{g 0} / T_{1}$ as a function of the heat coming from the gas at the surface, for different values of the exponent of the temperature dependence of the product $\rho_{R} \lambda_{R}$. 


$$
\ldots \frac{\rho_{g} \lambda_{g}}{\left(\rho_{g} \lambda_{g}\right)_{1}}=\left(\frac{T_{g}}{T_{1}}\right)^{w}=\theta^{w}
$$

eqn (A2) becomes

$$
2 \eta \frac{\partial \theta}{\partial \eta}+\frac{\partial}{\partial \eta}\left(\theta^{\omega} \frac{\partial \theta}{\partial \eta}\right)=0
$$

with boundary conditions $\theta(0)=1, \partial \theta /\left.\partial \eta\right|_{s}$ fixed. A Runge-Kutta integration scheme has been used to solve eqn (A5) for different values of $w$. In Fig. 5 the ratio $T_{80} / T_{1}$ is plotted as a function of $\partial \theta /\left.\partial \eta\right|_{s}$. To use this figure for some given conditions, give values to $T_{1}$ to obtain from Fig. $5 T_{g 0}$ as a function of $T_{1}$. Once this curve is known, enter with the given values of $T_{g 0}$ to derive $T_{1}$. The case $w=0$ corresponds to $\rho_{g} \lambda_{g}$ independent of temperature and in this case the classical result given in eqn (13) is recovered.

In general the parameter $\Gamma$ should be taken as

$$
\Gamma=\frac{T_{1}-T_{0}}{T_{g 0}-T_{3}}
$$

where $T_{1}$ is the correct jump temperature derived by the method outlined in the previous paragraph.

These results may be easily generalized to include an arbitrary dependence of the heat capacity on temperature by defining a new dependent variable.

$$
\phi=\int_{1}^{T_{g} / T_{1}} \frac{\rho_{g} \lambda_{g}}{\left(\rho_{g} \lambda_{g}\right)_{1}} \mathrm{~d}\left(T_{g} / T_{1}\right)
$$

It is also possible to account for variable properties in the solid.

\section{Appendix A}

\section{Nomenclature}

$B$ pre-exponential factor

c specific heat at constant pressure

$D_{g}$ binary diffusion coefficient

$E$ activation energy

Le Lewis number

$m$ stoichiometric coefficient

$n$ reaction order with respect to oxidizer mass fraction

$P$ nondimensional parameter defined by $\alpha \Gamma(1+\Delta$

$\Gamma)$ in the condensed phase and by $\alpha r(1+\eta$

$\Gamma) / \Gamma$ in the gas phase.

$Q$ heat evolved in surface reaction per unit mass $\lambda$ of fuel

$R$ Universal gas constant

$r$ ratio specific heats $c_{g} / c$

$T$ temperature

$t$ time

$v$ surface regression rate

$w$ exponent of the dependence of $\rho_{g} \lambda_{g}$ on $\psi$ temperature $x \quad$ space coordinate

$\boldsymbol{Y}$ oxidizer mass fraction

$y$ reduced mass fraction defined in eqn (23)

$z$ reduced space coordinate defined in eqn (24)

$\alpha$ dimensionless inverse heat of reaction $c\left(T_{g 0}-\right.$ $\left.T_{1}\right) / Q$

$\beta$ dimensionless activation energy defined in eqn

$\Gamma \quad$ ratio of thermal responsivities $V\left((\rho \lambda c)_{g} /(\rho \lambda c)\right)$

dimensionless parameter $\alpha(r+\Gamma)$

similarity variable defined in eqn (A1)

dimensionless temperature $\left(T-T_{1}\right) /\left(T_{g 0}-T_{1}\right)$ thermal conductivity

$\xi$ dimensionless space coordinate defined in eqn

$\rho$ density

$\sigma$ reduced temperature defined in eqn (24)

$\tau$ dimensionless time defined in eqn (12)

$\chi$ reduced temperature defined in eqn (22)

mass coordinate $\int_{0}^{x} \rho_{g} \mathrm{~d} x$ 